\title{
Interstitial and Cornual Ectopic Pregnancy: Conservative Surgical and Medical Management
}

\author{
Mamta Dagar $^{1}$ (D) Mala Srivastava $^{1} \cdot$ Indrani Ganguli $^{1} \cdot$ \\ Punita Bhardwaj $^{1} \cdot$ Nidhish Sharma $^{1} \cdot$ Deepak Chawla $^{1}$
}

Received: 11 October 2017/Accepted: 6 November 2017/Published online: 28 November 2017

(C) Federation of Obstetric \& Gynecological Societies of India 2017

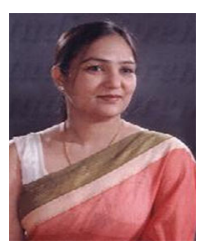

\begin{abstract}
About the Author
Dr. Mamta Dagar is MD (Ob-Gyn), MRCOG, FICOG, FICMCH, trained in Gynae Laparoscopy from Kiel, Germany and Robotic Surgery at EEC, Paris. She is graduate and postgraduate of prestigious Institute Lady Hardinge Medical College. She is currently working as a Consultant in Institute of Obstetrics and Gynaecology at Sir Ganga Ram Hospital, Delhi and Associate Professor GRIPMER, with more than 20 year of clinical experience. She is the Course Convener for AICC-RCOGNZ Colposcopy Courses and Member Representative RCOG-NZ India. She is a keen academician with publications in various peer reviewed journals. She has been co-investigator in several research projects with DST, DBT and ICMR. Dr. Dagar is an active member of RCOG-NZ, teaching faculty for MRCOG courses and is Editor, IMS Delhi Chapter.
\end{abstract}

\begin{abstract}
Background Interstitial and cornual ectopic pregnancy is rare, accounting for $2-4 \%$ of ectopic pregnancies and remains the most difficult type of ectopic pregnancy to diagnose due to low sensitivity and specificity of symptoms
\end{abstract}

Dr. Mamta Dagar is a Associate Prof. GRIPMER \& Consultant (ObGyn) of Sir Ganga Ram Hospital, Delhi. Dr. Mala Srivastava is a Professor, Senior Consultant, Obstetrics and Gynaecology, Sir Ganga Ram Hospital, Delhi. Dr. Indrani Ganguli is a Professor, HOD, Senior Consultant, Obstetrics and Gynaecology Department, Sir Ganga Ram Hospital, Delhi. Dr. Punita Bhardwaj is a Senior Consultant, Sir Ganga Ram Hospital, Delhi. Dr. Nidhish Sharma is a Consultant, Sir Ganga Ram Hospital, Delhi. Dr. Deepak Chawla is a Senior Consultant, Sir Ganga Ram Hospital, Delhi.

Mamta Dagar

drmamtadagar@gmail.com

1 Sir Ganga Ram Hospital Marg, Rajinder Nagar, New Delhi 110060, India and imaging. The classic triad of ectopic pregnancy-abdominal pain, amenorrhea and vaginal bleeding-occurs in less than $40 \%$ of patients. The site of implantation in the intrauterine portion of fallopian tube and invasion through the uterine wall make this pregnancy difficult to differentiate from an intrauterine pregnancy on ultrasound. The high mortality in this type of pregnancy is partially due to delay in diagnosis as well as the speed of hemorrhage.

Methods Three cases of interstitial pregnancy were retrospectively analyzed.

Result Successful laparoscopic cornuostomy and removal of products of conception were performed in two cases, while one case was successfully managed by local injection with KCL and methotrexate followed by systemic methotrexate.

Conclusion Early diagnosis and timely management are key to the management of interstitial and cornual ectopic pregnancy. With expertise in ultrasound imaging and advances in laparoscopic skills progressively, conservative 
medical and surgical measures are being used to treat interstitial and cornual ectopic pregnancy successfully.

Keywords Interstitial pregnancy · Cornual pregnancy · Laparoscopic cornuostomy · Methotrexate

\section{Introduction}

Interstitial and cornual pregnancy is a rare and most dangerous form of ectopic pregnancy, accounting for 2-4\% of all ectopic pregnancies [1]. Clinician often uses the term cornual ectopic pregnancy interchangeably with interstitial pregnancy. By definition, a cornual pregnancy refers to the implantation and development of a gestational sac in one of the upper and lateral portions of the uterus. Conversely, an interstitial pregnancy is a gestational sac that implants within the proximal, intramural portion of the fallopian tube that is enveloped by the myometrium [2, 3]. The interstitial portion is approximately $0.7 \mathrm{~mm}$ in width and approximately $1-2 \mathrm{~cm}$ in length. The surrounding myometrial tissue allows progression of the pregnancy into the second trimester, but rupture at such an advanced gestation may result in catastrophic hemorrhage with a mortality of up to $2 \%$ [1].

Interstitial and cornual pregnancy remains the most difficult type of ectopic pregnancy to diagnose due to low sensitivity and specificity of symptoms and imaging. The classic triad of ectopic pregnancy abdominal pain, amenorrhea and vaginal bleeding occurs in $<40 \%$ of patients. The site of implantation in the intrauterine portion of fallopian tube and invasion through the uterine wall makes this pregnancy difficult to differentiate from an intrauterine pregnancy on ultrasound. The ultrasonographic criteria proposed for diagnosing this condition are: an empty uterine cavity, a gestational sac located eccentrically and $>1 \mathrm{~cm}$ from the most lateral wall of the uterine cavity, and a thin $(<5 \mathrm{~mm})$ myometrial layer surrounding the gestational sac [4]. "Interstitial line sign" that extends from the upper region of the uterine horn to border the intramural portion of the fallopian tube has also been used [5]. Besides this, three-dimensional ultrasonography scans and magnetic resonance imaging allow for accurate early diagnosis of interstitial pregnancy if suspected on two-dimensional ultrasonography scans [6, 7].

Traditionally, treatment of interstitial pregnancy has been surgical and may include hysterectomy or cornual resection by laparotomy or laparoscopy [1]. But increasingly, more conservative approaches are being used such as cornuostomy instead of cornual resection, as well as laparoscopy in place of laparotomy. In the last few years, the use of more conservative surgical alternatives, such as cornuostomy rather than cornuectomy, is introduced to better preserve uterine integrity for future fertility. Many cases of laparoscopic cornuostomy have been reported in the literature so far [8,9].

Although patients with serious signs and symptoms of ectopic pregnancy (hypotension, severe abdominal pain or heavy vaginal bleeding) are likely to receive immediate surgical intervention, those with milder symptoms may be considered for medical therapy (methotrexate).

We report here three cases of interstitial and cornual ectopic pregnancy of which two were successfully managed by laparoscopic cornuostomy, while one was managed with local injection of $\mathrm{KCl}$ and methotrexate followed by systematic methotrexate.

\section{Case Report}

\section{Case I}

A 23-year-old woman, third gravida with previous two vaginal deliveries, referred to our hospital at $7+3$-week gestation as a suspected case of cornual ectopic pregnancy. She had earlier bleeding at $5+2$-week gestation, and pelvic USG done outside suggested left cornual ectopic pregnancy and was given injection methotrexate $75 \mathrm{mg}$ intramuscularly. There was no history of tuberculosis (TB), pelvic inflammatory disease (PID) or surgery. On admission, she was hemodynamically stable. Abdominal examination was normal. On per vaginum examination os was closed with minimal bleeding and no cervical exitation pain. Uterus was retroverted, para size with minimal left fornix fullness. On admission, her $\beta$-hCG was 9169 IU/1 and USG pelvis suggested a heteroechoic well-defined rounded area $(3.5 \times 3.3 \mathrm{~cm})$ with peripheral thick echogenic rim (trophoblast) and central cystic area $11.9 \mathrm{~mm}$ corresponding to gestational age 6 weeks 1 day with tiny yolk sac in the left-sided cornu of uterus, with surrounding myometrium $3 \mathrm{~mm}$ (Fig. 1).

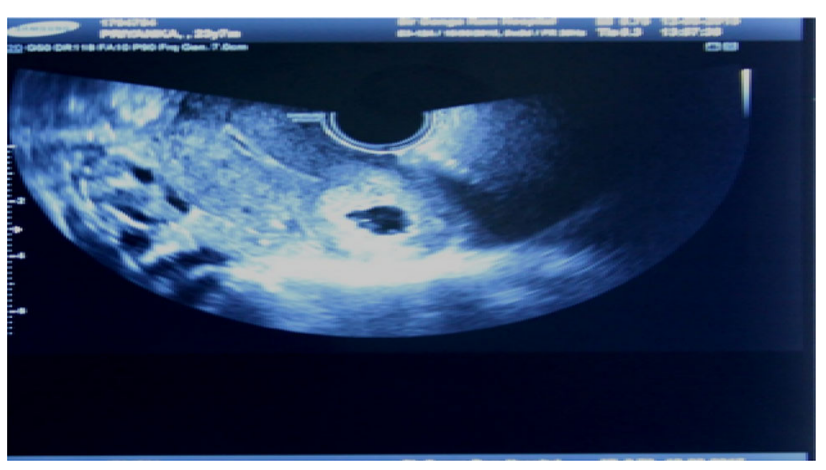

Fig. 1 Transvaginal two-dimensional ultrasonography scan in transverse view showing left interstitial ectopic pregnancy in case 1 


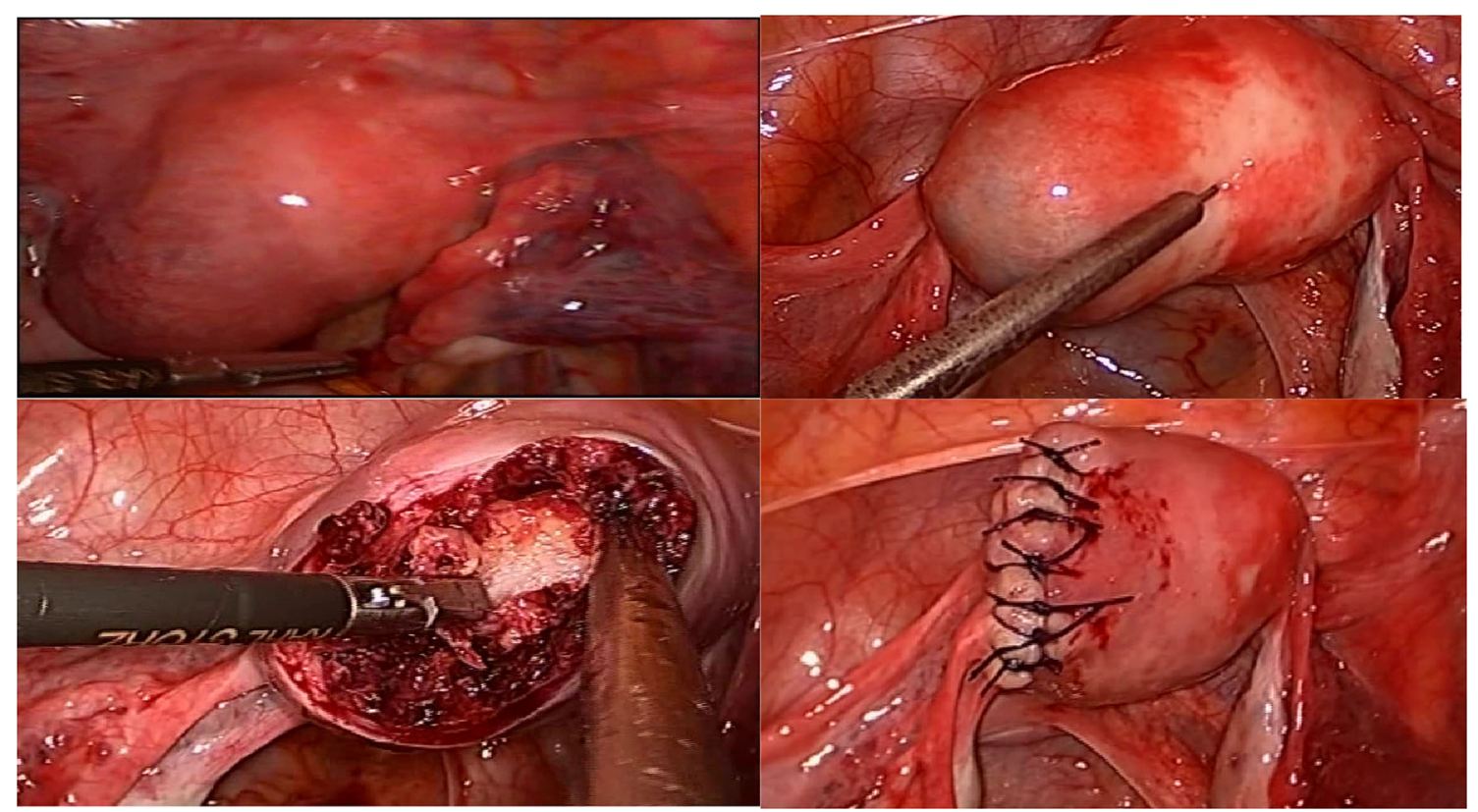

Fig. 2 Case 1; (i) laparoscopic view of left interstitial ectopic pregnancy; (ii) after injection of diluted vasopressin, (iii) removal of POCs after incision, (iv) final result of surgery, interrupted sutures

Medical managment was considered initially in view of hemodynamic stability after informed consent and serial S. $\beta$-hCG on day 0,4 and 7 was 9169,5410 and 5561 IU/1 respectively. In view of failed medical management decision for surgical intervention was taken. She underwent laparoscopic left cornuostomy. The uterus was injected with diluted vasopressin (20 in $100 \mathrm{ml}$ of normal saline) and uterine bulge was incised with scissors, and products of conception and gestational sac were removed followed by repair of incision with Vicryl no 1. Post-op period was uneventful. Histopathology report confirmed products of gestation (ectopic cornual gestation). Serial decline in $\beta$ hCG noted till 6 weeks when it became $<2$ IU/l. Patient was advised contraception COCP at 6-week follow-up and counseled regarding the risk of ruptured uterus in the next pregnancy and elective cesarean in future pregnancy (Fig. 2).

\section{Case II}

A 39-year-old woman, gravida 4, para 1 previous LSCS with previous 2 miscarriages presented with amenorrhea $6+3$ weeks and no other complaints. She was hemodynamically stable. Per vaginum examination revealed bulky uterus with no fornicial mass or tenderness. Transvaginal ultrasound suggested heteroechoic mass $2.9 \times 2.5 \times 2.8 \mathrm{~cm}$ in right uterine cornu with increased vascularity and small cystic component of size $6 \times 3 \mathrm{~mm}$ within gestational sac indicative of right cornual ectopic pregnancy. She was counseled and she opted for conservative surgical management. She underwent laparoscopic right cornuostomy after informed consent. The uterus was injected with diluted vasopressin (20 in $100 \mathrm{ml}$ of normal saline) and uterine bulge was incised with scissors, and products of gestation were removed followed by repair of incision with Vicryl no 1. Histopathology report confirmed cornual ectopic pregnancy with products of gestation. She was followed with serial weekly S. $\beta$-hCG till it became $<2.0 \mathrm{IU} / \mathrm{l}, 4$ weeks later (Fig. 3).

\section{Case III}

A 28-year-old woman second gravida with previous missed miscarriage presented at $9+4$-week gestation with pelvic USG suggestive of right-sided cornual ectopic pregnancy.

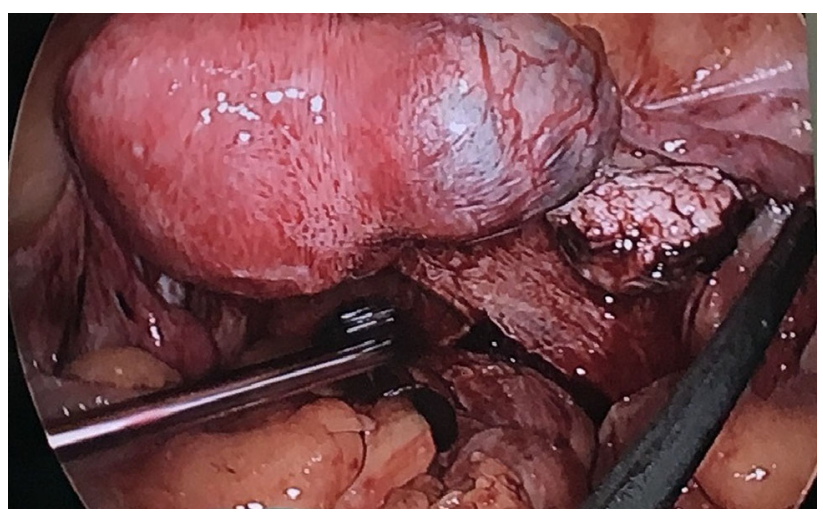

Fig. 3 Laproscopic view of right interstitial ectopic pregnancy in case 2 


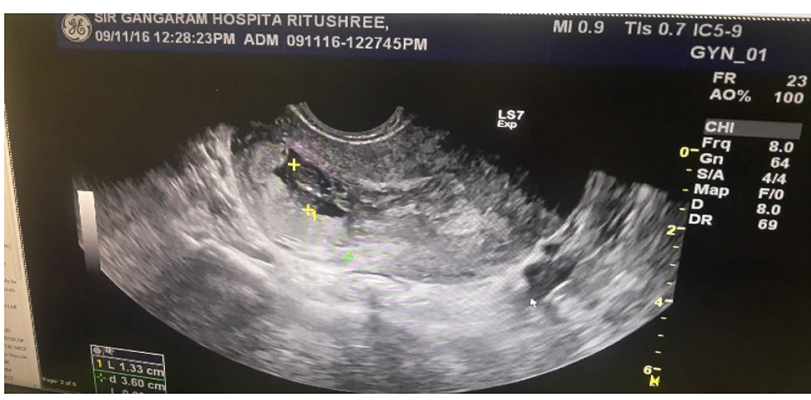

Fig. 4 Transvaginal two-dimensional ultrasonography scan in transverse view showing right-sided cornual ectopic pregnancy

She was under strict follow-up since 7-week gestation as no clear-cut diagnosis of cornual ectopic pregnancy was made on ultrasound. On admission, she was hemodynamically stable. Per abdominal examination was normal. On per vaginal examination, uterus was retroverted, bulky, soft, with no cervical motion tenderness and no adnexal mass was felt. USG pelvis was suggestive of gestational sac of $8+5$ weeks with cardiac activity, CRL $20.7 \mathrm{~mm}$, myometrium thickness $3.6 \mathrm{~mm}$ suggestive of cornual ectopic pregnancy. Her S. $\beta$-hCG was 51,569 IU/l. Management options both medical and surgical were discussed. She opted for medical management. She received transvaginal USGguided intra-amniotic injection of $\mathrm{KCl}(4 \mathrm{ml})$ and intraplacental injection of methotrexate $(25 \mathrm{mg})$ after informed consent. Disappearance of cardiac activity was noted on USG. She received injection methotrexate $50 \mathrm{mg}$ intramuscularly after $24 \mathrm{~h}$ followed by injection folinic acid $5 \mathrm{mg}$ next day. She remained stable during hospital stay and was discharged in stable condition after 5 days.

Follow-up was done with weekly serum beta hCG till it became $<2$ IU/l and pelvic USG. Subsequent decline in serum beta hCG and reduction in size of ectopic gestational sac were noted on USG (Fig. 4).

\section{Discussion}

Early recognition of the interstitial and cornual pregnancy is essential. Diverse therapeutic options exist for the management of this condition. Managing an interstitial pregnancy is dependent upon whether the ectopic pregnancy has ruptured and the stability of the patient. Ultrasonography and a high index of suspicion have allowed for early diagnosis and increased success of conservative management for interstitial ectopic pregnancy.

Traditionally, the treatment of interstitial and cornual pregnancy has been laparotomy, cornual resection or hysterectomy in cases presenting with hypovolemic shock and ruptured uterus [10, 11]. However, in hemodynamically stable patients, conservative measures may be attempted including medical management and laparoscopy [12]. In a report by Tulandi and Al-Jaroudi [13], the management of 32 cases of interstitial pregnancy was discussed. Eight women were treated with methotrexate either systemically $(n=4)$, locally under ultrasonographic guidance $(n=2)$ or under laparoscopic guidance $(n=2)$. Eleven patients were treated by laparoscopy and 13 by laparotomy. Systemic methotrexate treatment failed in three patients, and they subsequently required surgery. Persistently elevated serum $\beta$-hCG levels were found in one patient after laparoscopic cornual excision, and she was successfully treated with methotrexate. Subsequent pregnancy was achieved in ten patients. No uterine rupture was encountered during pregnancy or labor [13].

In a prospective observational study [14] at St George's Hospital Medical School in London, 17 out of 20 women with cornual pregnancy were treated with single-dose intramuscular methotrexate, which was administered on day 0 . A second dose of methotrexate was given if the human chorionic gonadotrophin (hCG) levels had not fallen by $15 \%$ between days 4 and 7 . Sixteen $(94 \%)$ were treated successfully, including all four cases with presence of fetal heart activity. A second methotrexate dose was given to six women.

Where surgery can be avoided, systemic medical management is a safe and considered to be effective treatment for cornual pregnancy. In the event the interstitial pregnancy is medium-sized $(<5 \mathrm{~cm})$, conservative management with methotrexate is often used with caution [15]. Unfortunately, methotrexate treatment has been associated with a $9-65 \%$ failure rate $[16,17]$.

Systemic administration of methotrexate is widely used in nearly all forms of ectopic pregnancy in patients with stable vital signs. However, transvaginal USG-guided local methotrexate injection to the cornual gestational sac has been presented as case reports and suggested to be a safe and effective alternative to surgical and systemic methotrexate therapy [18]. Especially in live ectopic gestations, systemic methotrexate should not be the first-line treatment due to high failure rates $(30 \%)$, and surgery or local methotrexate injection into the sac should be considered [19]. In case 3, we presented the successful combined use of systemic and transvaginal USG-guided local $\mathrm{KCl}$ and methotrexate injection in the management of a cornual pregnancy with a very high initial beta hCG level and live ectopic.

In case 3, we combined both modalities, local and systemic methotrexate administration along with local $\mathrm{KCl}$ injection, which is one the few case reports of such an approach. In the literature, successful treatment of cornual pregnancy with just a single dose of methotrexate has been reported [20, 21]. Medical treatment with a single methotrexate injection has been recommended as an 
alternative to surgical treatment of cornual pregnancy. However, it is associated with significantly increased risk of failure, subsequent uterine rupture and emergency surgery [21]. Cornual pregnancy with an initial beta hCG value of $<5000 \mathrm{IU} / \mathrm{ml}$ is usually treated successfully with single-dose methotrexate, but when the value is $>5000 \mathrm{IU} / \mathrm{ml}$, more than a single dose is usually required and failure or complication is more likely [22]. However, upper limit of beta hCG value at which medical treatment with methotrexate will fail is not clear. For local administration of methotrexate, various injected doses such as $12.5,25$ and $100 \mathrm{mg}$ were given in the literature.

Regarding surgical treatment, main concerns are hemorrhage and the need for adequate cornual reconstruction to prevent uterine rupture in future subsequent pregnancy, which necessitate advanced laparoscopic skills and technique $[23,24]$. Integrity of myometrium after conservative treatments is unclear; patients must be counseled carefully about the risk of uterine rupture in subsequent pregnancy. To prevent this severe complication, normal uterine tissue must be preserved, avoiding tissue damage by electrosurgery and by minimal excision of cornual tissue [24, 25]. Suture closure of the cornual defect remains the most appropriate method for the achievement of hemostasis and may also minimize the risk of uterine rupture in subsequent pregnancy $[25,26]$.

To minimize blood loss, different techniques such as uterotonic vaginal misoprostol insertion and laparoscopic injection of vasoconstrictors prior to surgery can be used. Diluted intra-myometrial vasopressin injection is the most well-known and preferable procedure [27, 28] obtaining a fast whitening of the tissue and allowing a non-bleeding surgery effectively. Other methods used by laparoscopists include ligation of ascending branches of the uterine vessels, uterine de-vascularization, apposition of fibrin glue or tourniquets applied around the cornual mass or through the avascular area of the broad ligament to minimize bleeding [26, 29]. These methods require a high level of laparoscopic skills [29]. We used diluted vasopressin injection to minimize bleeding.

Close antenatal follow-up of patient with history of cornual ectopic pregnancy (CEP) is mandatory. The incidence of recurrent CEP is unknown [30, 31]. Transvaginal ultrasound should be performed 5-6 weeks after the last menstrual period to ensure the correct implantation of the subsequent gestation [31]. Typically, elective cesarean section should be planned to reduce the risk of rupture in labor $[25,31]$.

\section{Conclusion}

Early diagnosis and timely management is the key to management of interstitial and cornual ectopic pregnancy. With expertise in ultrasound imaging and advances in laparoscopic skills progressively, conservative medical and surgical measures are being used to treat interstitial and cornual ectopic pregnancy successfully.

In conclusion, combined use of systemic and transvaginal USG-guided local methotrexate injection along with $\mathrm{KCl}$ in the management of cornual pregnancy is a safe and effective treatment method in hemodynamically stable patients even in the presence of an initial high beta hCG value and cardiac activity. Therefore, it might be considered as an initial therapeutic option in unruptured cornual pregnancy before proceeding to surgical intervention.

\section{Compliance with Ethical Standards}

Conflict of interest The authors declare that they have no conflict of interest.

Ethical Approval This article does not contain any studies with human participants or animals performed by any of the authors.

Informed Consent For this type of study formal consent is not required.

\section{References}

1. Faraj R, Steel M. Management of cornual (interstitial) pregnancy. Obstet Gynaecol. 2007;9:249-55.

2. Malinowski A, Bates SK. Semantics and pitfalls in the diagnosis of cornual/interstitial pregnancy. Fertil Steril. 2006;86(6):1764.e11-4.

3. Kun W, Tung W. On the lookout for rarity-interstitial/cornual pregnancy. Emerg Med J. 2001;8:147-50.

4. Timor-Tritsch IE, Monteagudo A, Materna C, et al. Sonographic evolution of cornual pregnancies treated without surgery. Obstet Gynecol. 1992;79:1044-9.

5. Ackerman TE, Levi CS, Dashefsky SM, et al. Interstitial line: sonographic finding in interstitial ectopic pregnancy. Radiology. 1993;189:83-7.

6. Filhastre M, Dechaud H, Lesnik A, et al. Interstitial pregnancy: role of MRI. Eur Radiol. 2005;15(1):93-5.

7. Araujo Junior E, Zanforlin Filho SM, Pires CR, et al. Three dimensional transvaginal sonographic diagnosis of early and asymptomatic interstitial pregnancy. Arch Gynecol Obstet. 2007;275(3):207-10.

8. Chan LY, Yuen PM. Successful treatment of ruptured interstitial pregnancy with laparoscopic surgery. A report of 2 cases. J Reprod Med. 2003;48(7):569-71.

9. Moon HS, Choi YJ, Park YH, et al. New simple endoscopic operations for interstitial pregnancies. Am J Obstet Gynecol. 2000;182:114-21.

10. Katz DL, Barrett JP, Sanfilippo JS, et al. Combined hysteroscopy and laparoscopy in the treatment of interstitial pregnancy. Am J Obstet Gynecol. 2003;188:1113-4.

11. Brewer H, Gefroh S, Bork M, et al. Asymptomatic rupture of a cornual ectopic in the third trimester. J Reprod Med. 2005;50(9):715-8.

12. Bernstein HB, Thrall MM, Clark WB. Expectant management of intramural ectopic pregnancy. Obstet Gynecol. 2001;97:826-7.

13. Tulandi T, Al-Jaroudi D. Interstitial pregnancy: results generated from the Society of Reproductive Surgeons' Registry. Obstet Gynecol. 2004;103(1):47-50. 
14. Jermy K, Thomas J, Doo A, et al. The conservative management of interstitial pregnancy. BJOG. 2004;111:1283-8.

15. Tanaka T, Hayashi $H$, Kutsuzawa $T$, et al. Treatment of interstitial ectopic pregnancy with methotrexate: report of a successful case. Fertil Steril. 1982;37:851-2.

16. Tulandi T, Al-Jaroudi D. Interstitial pregnancy: results generated from the society of reproductive surgeons registry. Obstet Gynecol. 2004;103:47-50.

17. Tang A, Baartz D, Khoo SK. A medical management of interstitial ectopic pregnancy: a 5-year clinical study. Aust N Z J Obstet Gynaecol. 2006;46(2):107-11.

18. Hafner T, Aslam N, Ross JA, et al. The effectiveness of nonsurgical management of early interstitial pregnancy: a report of ten cases and review of the literature. Ultrasound Obstet Gynaecol. 1999;13(2):131-6.

19. Monteagudo A, Minior VK, Stephenson C, et al. Non-surgical management of live ectopic pregnancy with ultrasound guided local injection-a case series. Ultrasound Obstet Gynecol. 2005;25(3):282-8.

20. Dilbaz S, Demir B, Dilbaz B. Treating cornual pregnancy with a single methotrexate injection-a case report of 3 cases. J Reprod Med Obstet Gynecol. 2005;50(2):141-4.

21. Gherman RB, Stitely M, Larrimore C, et al. Low dose methotrexate treatment for interstitial pregnancy: a case report. J Reprod Med Obstet Gynecol. 2000;45(2):142-4.

22. Jermy K, Thomas J, Doo A, et al. The conservative management of interstitial pregnancy. Int $J$ Obstet Gynaecol. 2004;111:1283-8.

23. Mahmoud MS. Technique for the laparoscopic management of a cornual ectopic pregnancy. J Minim Invasive Gynecol. 2016;23:300.
24. Moon HS, Kim SG, Park GS, et al. Efficacy of bleeding control using a large amount of highly diluted vasopressin in laparoscopic treatment for interstitial pregnancy. Am J Obstet Gynecol. 2010;203(1):30.e1-6.

25. Ng S, Hamontri S, Chua I, et al. Laparoscopic management of 53 cases of cornual ectopic pregnancy. Fertil Steril. 2009;92:448-52.

26. Hwang JH, Lee JK, Lee NW, et al. Open cornual resection versus laparoscopic cornual resection in patients with interstitial ectopic pregnancies. Eur J Obstet Gynecol Reprod Biol. 2011;156:78-82.

27. Pluchino N, Ninni F, Angioni S, et al. Spontaneous cornual pregnancy after homolateral salpingectomy for an earlier tubal pregnancy: a case report and literature review. J Minim Invasive Gynecol. 2009;16:208-11.

28. Nirgianakis K, Papadia A, Grandi G, et al. Laparoscopic management of ectopic pregnancies: a comparison between interstitial and "more distal" tubal pregnancies. Arch Gynecol Obstet. 2016;295:95-101.

29. Choi YS, Eun DS, Choi J, et al. Laparoscopic cornuotomy using a temporary tourniquet suture and diluted vasopressin injection in interstitial pregnancy. Fertil Steril. 2009;91:1933-7.

30. Siow A, Ng S. Laparoscopic management of 4 cases of recurrent cornual ectopic pregnancy and review of literature. J Minim Invasive Gynecol. 2011;18:296-302.

31. Moawad NS, Mahajan ST, Moniz MH, et al. Current diagnosis and treatment of interstitial pregnancy. Am J Obstet Gynecol. 2010;201:15-29. 